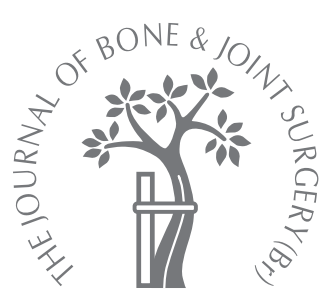

\title{
Anatomical variations in vastus medialis obliquus and its implications in minimally- invasive total knee replacement
}

\author{
AN MRI STUDY
}

\author{
V. I. Roberts, \\ P. K. R. Mereddy, \\ N. J. Donnachie, \\ S. Hakkalamani \\ From Arrowe Park \\ Hospital, Upton, \\ England
}

- V. I. Roberts, MRCS, Research Fellow in

Orthopaedics

Division of Orthopaedic Surgery

Glenfield Hospital, Groby Road, Leicester LE3 90P, UK.

In. K. R. Mereddy, MS(Orth), DNB(Orth), MRCSEd, Research Fellow in Orthopaedics

N. J. Donnachie, FRCS(Orth), Consultant Orthopaedic

Surgeon

Arrowe Park Hospital, Upton, Wirral, Merseyside CH49 5PE, UK.

In. Hakkalamani, MS(Orth), MRCSEd, Specialist Registrar in Orthopaedics

Countess of Chester Hospital, Liverpool Road, Chester,

Cheshire CH1 1UL, UK.

Correspondence should be sent to Ms V. I. Roberts; e-mail: Vero_robertsl@hotmail.com

(C2007 British Editorial Society of Bone and Joint Surgery doi:10.1302/0301-620X.89B11. $18636 \$ 2.00$

$J$ Bone Joint Surg $[\mathrm{Br}]$ 2007;89-B:1462-5.

Received 13 September 2006;

Accepted after revision 10 July 2007

The emergence of minimally-invasive total knee replacement (TKR) has led to the refinement of several surgical approaches, including the quadriceps-sparing and subvastus. There is some disagreement as to the precise definition of the term 'minimallyinvasive', especially in regard to the preservation of vastus medialis obliquus (VMO). It is known that the termination of VMO is variable and may make these approaches difficult. We have attempted to assess the factors influencing the insertion of VMO and the impact which they have on the approach for TKR.

The MR scans of 198 knees were examined to assess the variation in the insertion of VMO in relation to the patella and the effect of variables such as age, gender and the presence of concurrent osteoarthritis of the knee on the insertion.

Our findings showed that both age and the presence of osteoarthritis were contributing factors to changes in the level of insertion of VMO. Therefore, not all capsular incisions which extend proximal to the midpole of the patella will violate the quadriceps tendon.

There are many options when considering the surgical approach for total knee replacement (TKR). In 2005, of the 59037 TKRs recorded on the National Joint Registry of England and Wales, ${ }^{1} 54489$ had a medial parapatellar approach, 1072 a lateral parapatellar approach, 1100 a subvastus approach and the remainder other approaches such as the midvastus, intravastus and trivector.

In recent years, minimally-invasive TKR has been used increasingly. Several approaches have been refined to allow this development, including the subvastus and quadricepssparing approaches. Both aim to position the arthrotomy incision in such a way that the patella can be retracted without violation of the quadriceps tendon or of vastus medialis.

In the subvastus approach, the arthrotomy is an inverted ' $\mathrm{L}$ '-shaped incision, which extends along the inferior border of vastus medialis obliquus (VMO) and requires the muscle to be released from the periosteum to the level of the adductor tubercle. ${ }^{2}$ However, in some cases, this technique requires the division of the muscle fascia to allow adequate exposure and sufficient lateral mobility of the patella. ${ }^{3}$

In the quadriceps-sparing approach, the arthrotomy is described as beginning at the superomedial border of the patella where $\mathrm{VMO}$ inserts and ending $2 \mathrm{~cm}$ below the tibial joint line. ${ }^{4,5}$ Recently, it has been suggested that such an approach could not be considered quadriceps-sparing since it was found that any capsular incision which extended proximal to the midpole of the patella violated a portion of the quadriceps tendon. ${ }^{6}$

The insertion of VMO is variable. $\mathrm{Tria}^{7}$ stated that in most patients it is at, or proximal to, the superior pole of the patella, but others have found that the muscle belly may extend distal to the midpoint of the patella. ${ }^{6,8}$ This has led to a debate as to whether the quadriceps-sparing approach, and to some extent the subvastus approach, can truly be VMO-sparing and be considered to be minimally invasive. It is important to understand the anatomy of the knee, especially when there are obvious benefits to preserving the VMO. ${ }^{9}$ Compared with a standard TKR, a minimally-invasive procedure has been reported to have reduced blood loss, an improved pain score, a reduced hospital stay and an increased post-operative range of movement. ${ }^{9}$ Others, however, have reported no differences. ${ }^{10}$

Our aim was to clarify the anatomy of the medial side of the knee, particularly the insertion of VMO on the patella. We also determined the effect of age, gender, and the presence of concurrent osteoarthritis (OA) of the knee on the insertion. 


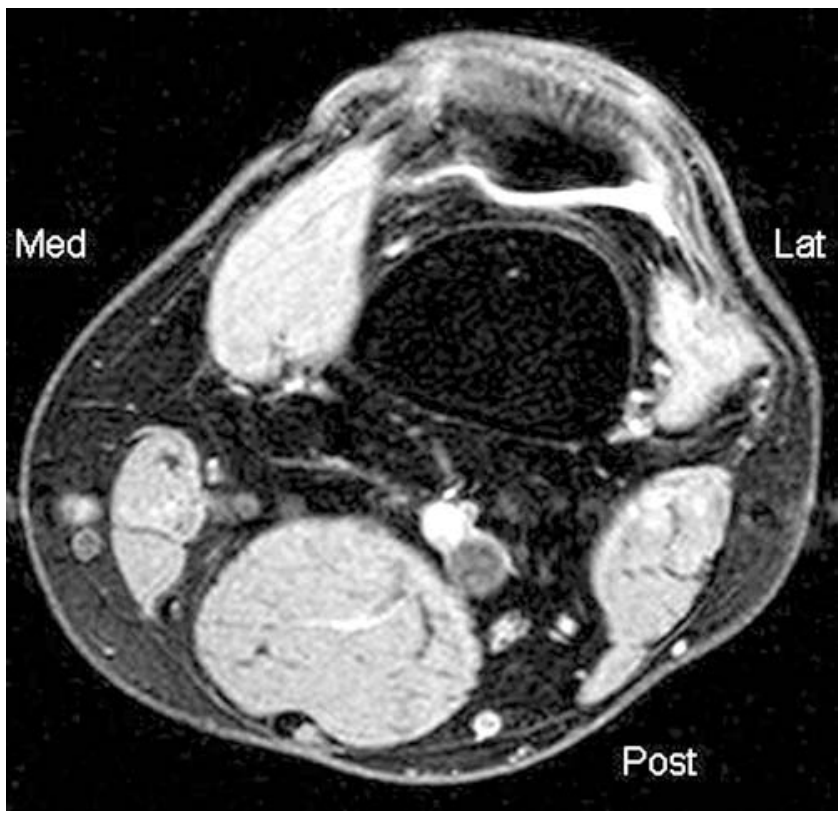

Fig. 1

MR scan showing the reference slice at the apex of the patella.

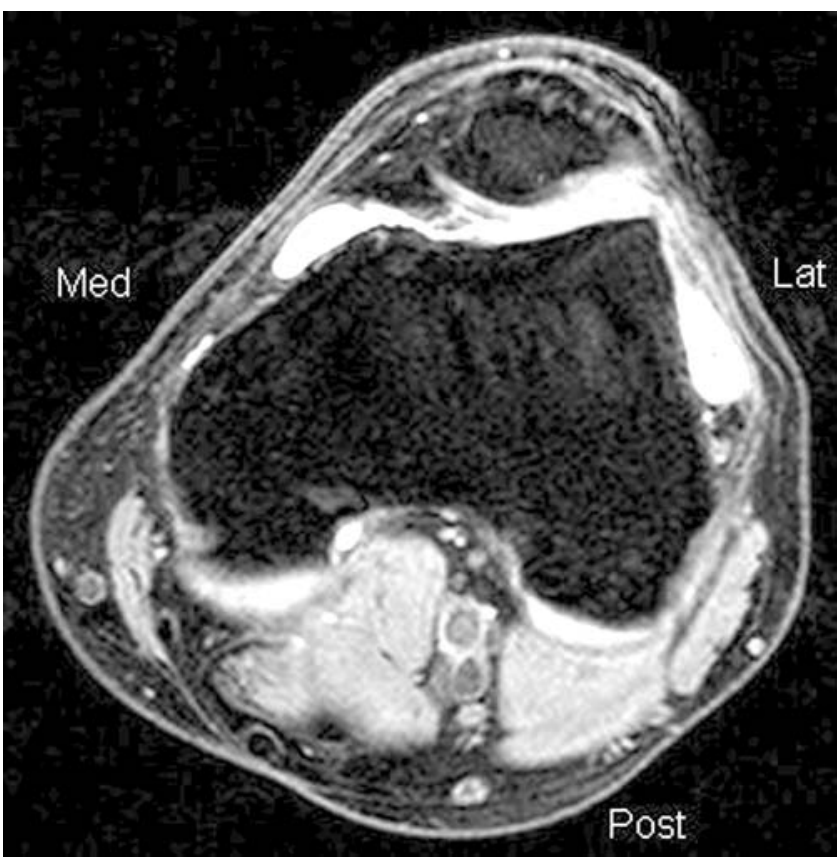

Fig. 2

MR scan showing the inferior border of patella.

\section{Patients and Methods}

The axial MR scans of 262 knees taken over a period of 12 months between January 2005 and December 2005 were

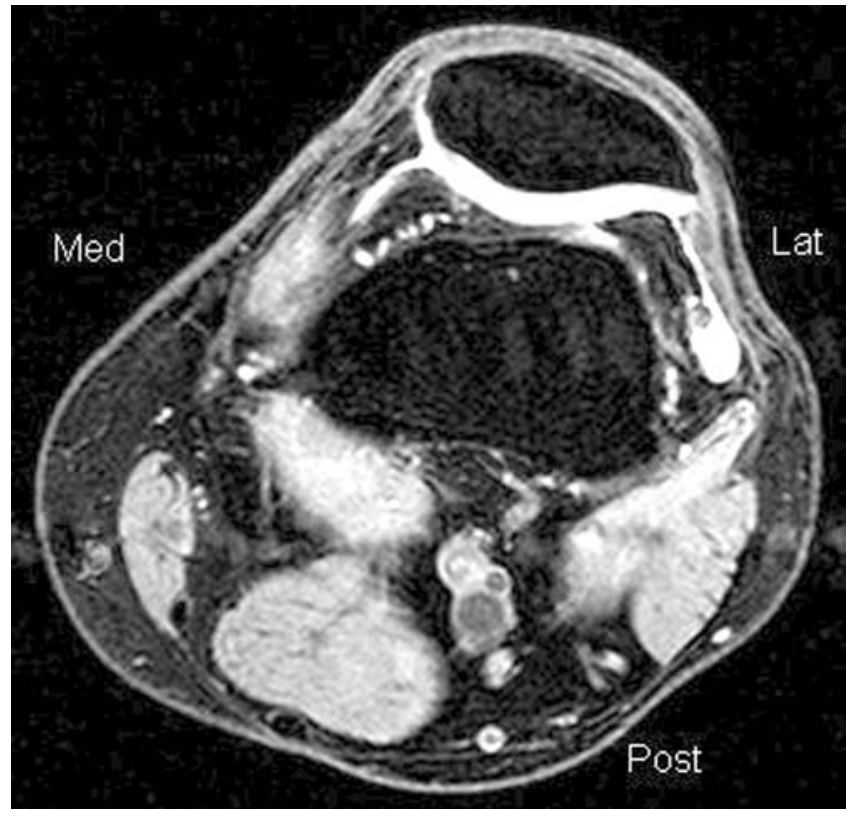

Fig. 3

Axial MR scan showing the presence of vastus medialis obliquus. examined. ${ }^{9}$ Of these, 38 were excluded in patients under the age of 16 years, those whose scans showed neoplastic pathology or were incomplete. In 26 patients, bilateral scans were available and only one was randomly selected for inclusion. The reasons for imaging the knee were not investigated. A total of 198 scans were analysed by two observers (VIR, PKRM). The radiographs of these patients were also assessed for the diagnosis of $\mathrm{OA}$ in the knee according to the Pridie classification. ${ }^{11}$

There were 106 females and 92 males with a mean age of 43 years (16 to 86 ). Of these, 134 were aged less than 50 years and 64 were older.

The MR scans were performed on a 1.5 Tesla machine (Philips Medical System, Noord, Netherlands) with all patients positioned supine. Axial three-dimensional (3D) water excitation sequences performed with a slice thickness of $4 \mathrm{~mm}$ were examined.

In order to calculate the patellar height, the apex of the patella was considered as the 'reference slice 1' (Fig. 1). The consecutive slices were followed distally to the last slice in which the patella was visible (Fig. 2). The muscle length of $\mathrm{VMO}$ was considered to be the length of the muscle from the apex of the patella and was measured by following it from reference slice 1 to the slice in which it was last visible (Figs 3 and 4). The patellar height and muscle length were calculated as the product of the number of MR slices and the thickness of the slice.

The ratio of the muscle length to patellar height (VMO:patella) was also calculated since this equates to the level of insertion of VMO on to the patella. The level of 


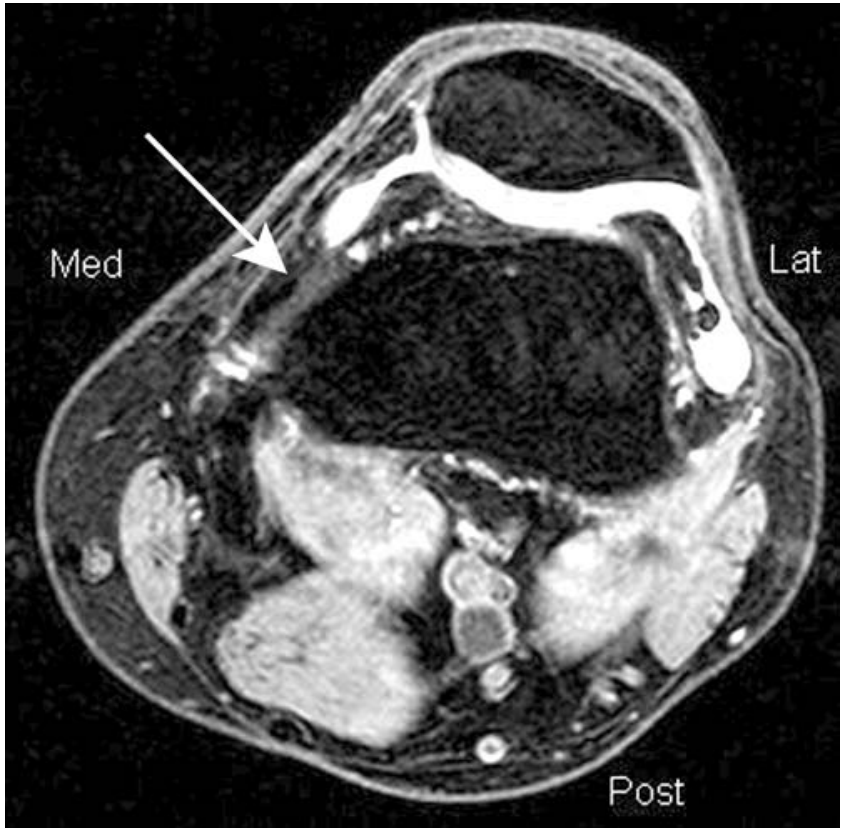

Fig. 4

Axial MR scan showing the absence of vastus medialis obliquus (arrow highlighting where the muscle was visible in the previous slice).

insertion was divided into two groups. Group 1 included those scans in which VMO terminated proximal to the midpoint of the patella (VMO:patella $<50 \%$ ) and group 2 those in which VMO terminated at, or distal to, the midpoint of the patella (VMO:patella $\geq 50 \%$ ).

In order to assess the intraobserver variation, each observer randomly selected 20 scans and blinded reassessment was done after eight weeks. In order to assess the interobserver variation, the 20 scans measured by the first observer (VIR) were randomly selected for blinded assessment by the second observer (PKRM).

Statistical analysis. This was done using SPSS version 14 (SPSS Inc., Chicago, Illinois). Non-parametric tests were used to calculate the significance. Statistical significance was set at a p-value $\leq 0.05$.

\section{Results}

With reference to the VMO:patella ratio, there were 119 patients $(60.1 \%)$ in group 1 , and $79(39.9 \%)$ in group 2 . The effect of the different variables on muscle length, patellar height and the level of insertion was determined.

Effect of gender. The mean height of the patella in women was $33.5 \mathrm{~mm}$ (24 to 44 ) and in men it was $37.8 \mathrm{~mm}$ (28 to 48 ). The mean VMO muscle length was $14.4 \mathrm{~mm}$ in women (4 to 28 ) and $16.8 \mathrm{~mm}$ in men (8 to 32 ).

There was a statistically significant difference in the relationships between both the height of the patella and VMO length and gender (Mann-Whitney $\mathrm{U}$ test, $\mathrm{p}<0.0001$ and $\mathrm{p}<0.0005$ respectively). The results showed that men had larger patellae and greater muscle length than women.

The mean VMO:patella ratio in women was $42.8 \%$ ( $13 \%$ to $71 \%$ ), and in men it was $44.8 \%$ ( $18 \%$ to $80 \%$ ). There was no statistically significant difference in the VMO:patella ratio between the genders (Mann-Whitney U test, $\mathrm{p}=0.2$ ).

Effect of age. Age had no impact on the height of the patella, with the mean patellar height being $35.3 \mathrm{~mm} \mathrm{(24} \mathrm{to}$ 48 ) in patients younger than 50 years and $35.2 \mathrm{~mm}$ (24 to 44 ) in those older than 50 years. It had a statistically significant effect on muscle length. The mean length was $16.4 \mathrm{~mm}$ (4 to 32 ) in patients under 50 years, and $13.7 \mathrm{~mm}$ ( 8 to 28 ) in those aged 50 years or more (Mann-Whitney U test, $\mathrm{p}<0.0005$ ).

Of the patients aged under 50 years, $68(50.7 \%)$ had a $\mathrm{VMO}$ which terminated in the proximal half of the patella. In the patients aged 50 years or older, $51(79.7 \%)$ showed this, which represented a statistically significant inverse relationship between the age of the patient and the VMO:patella ratio (Mann-Whitney U test, $\mathrm{p}<0.0001$ ).

However, there was no statistically significant relationship between the level of insertion of VMO and the gender of the patient in either age group (Mann-Whitney U test, $\mathrm{p}=0.339$ ).

Effect of osteoarthritis. Osteoarthritis was detected in the radiographs of 82 patients $(41.4 \%)$. A statistically significant relationship was noted between the presence of OA and the VMO length (Kruskal-Wallis test, $\mathrm{p}=0.001$ ). Patients with OA had a mean length of $14.2 \mathrm{~mm}$ (8 to 24) and those without a mean length of $16.5 \mathrm{~mm}$ (4 to 32) (Kruskal-Wallis test, $\mathrm{p}=0.001$ ). There was also a statistically significant relationship between $\mathrm{OA}$ and the VMO:patella ratio since patients with OA were found to have a more proximal insertion (Mann-Whitney U test, $\mathrm{p}<0.0005)$. In those with OA, the mean VMO:patella ratio was $39.9 \%$ (18\% to $60 \%$ ), and in those without it was $46.7 \%$ (13\% to $80 \%$ ).

As expected, there was a statistically significant relationship between the age of the patient and OA (Kruskal-Wallis test, $\mathrm{p}=0.001)$. However, there was no statistically significant difference between the gender of the patient and OA (Kruskal-Wallis test, $\mathrm{p}=0.54$ ).

Intra- and interobserver variation. There was no statistically significant difference in intra- and interobserver measurements (two-sample $t$-test, $\mathrm{p}=0.614, \mathrm{p}=0.530$, $\mathrm{p}=0.163$ and $\mathrm{p}=0.379$, respectively).

\section{Discussion}

The quadriceps-sparing and subvastus approaches are relatively new techniques which have aided the development of minimally-invasive TKR. With their emergence has come a new nomenclature, which has led to confusion and disagreement.

Our study was undertaken to clarify the anatomy of $\mathrm{VMO}$, and to assess factors influencing its termination and their impact on the approach for TKR. Our results showed 
that the point of insertion of $\mathrm{VMO}$ was variable and changed significantly with advancing age and the presence of $\mathrm{OA}$ of the knee.

There was no statistical difference in the level of insertion of $\mathrm{VMO}$ according to gender. However, there was a significant difference between the height of the patella and gender and between the length of the VMO muscle and gender. This may be related to the generally larger skeleton and muscle conformation of men compared with women.

Our results showed over $50 \%$ of patients aged under 50 years had a VMO which terminated at, or more distal, to the midpoint of the patella, whereas only $20 \%$ of patients aged 50 years or more had a similar termination. Therefore, only one-fifth of patients aged 50 years or more would be unlikely to be suitable for the quadricepssparing approach. Similar observations were reported by Bose, Kanagasuntheram and Osman ${ }^{12}$ in a cadaveric study.

These age-related changes in the insertion of VMO have not been described previously. In our study, there was a statistically inverse relationship between the level of termination of VMO and the age of the patient so that with increasing age, the termination of VMO became more proximal, probably because of muscle atrophy.

We observed a significant relationship between the length of $\mathrm{VMO}$ and the presence of $\mathrm{OA}$ and therefore between patients who had OA and the level of insertion of the muscle. This could be explained by the muscular wasting encountered in OA.

Recent studies have shown that MRI is useful in studying the anatomy of the knee. ${ }^{6,13}$ Limitations of our study were that the patients included had MRI performed for various indications. In addition, it was a retrospective analysis and no details of the height and weight of the patients studied were available to define the reasons for the fact that men had a larger patella and a longer VMO than women.

The insertion of $\mathrm{VMO}$ is variable and the contributing factors for the anatomical changes in VMO are the age of the patient and the presence of OA. Therefore not all cap- sular incisions which extend proximal to the midpoint of the patella will violate the quadriceps tendon, especially if the patient is aged over 50 years or has OA. However, the insertion of VMO can be distal to the midpoint of the patella in some patients, and could obscure the surgical view, ${ }^{4}$ or may even preclude the use of true minimallyinvasive techniques.

We wish to thank Dr P. Evans (Consultant Radiologist) and Mrs D. Collins (Superintendent Radiographer) of Arrowe Park Hospital, Upton, Wirral, UK for their contribution to this study. The authors would like to state that V. I. Roberts and P. K. R. Mereddy made an equal contribution to this paper.

No benefits in any form have been received or will be received from a commercial party related directly or indirectly to the subject of this article.

\section{References}

1. No authors listed. National Joint Register for Endgland and Wales. Third Annual Report, 2006. http://www.njr.centre.org.uk/documents/reports/annual/3rd/NJR_ AR2_LR.pdf (date last accessed 29 August 2007).

2. Hofmann AA, Plaster RL, Murdock LE. Subvastus (Southern) approach for primary total knee arthroplasty. Clin Orthop 1991;269:70-7.

3. Aglietti P, Baldini A, Sensi L. Quadriceps-sparing versus mini-subvastus approach in total knee arthroplasty. Clin Orthop 2006;452:106-11.

4. Alan RK, Tria AJ Jr. Quadriceps-sparing total knee arthroplasty using posterior stabilised TKA design. J Knee Surg 2006;19:71-7

5. Chen AF, Alan RK, Redziniak DE, Tria AJ Jr. Quadriceps sparing total knee replacement: the initial experience with results at two to four years. J Bone Joint Surg [Br] 2006;88-B:1448-53.

6. Pagnano MW, Meneghini RM, Trousdale RT. Anatomy of the extensor mechanism in reference to quadriceps-sparing TKA. Clin Orthop 2006;452:102-5.

7. Tria AJ Jr. Minimally invasive total knee arthroplasty using the quadriceps-sparing approach. In: Scuderi G, Tria AJ Jr, Berger RA, eds. MIS techniques in orthopaedics. New York: Springer-Verlag, 2005:349-66.

8. Peeler J, Cooper J, Porter MM, Thliverai JA, Anderson JE. Structural parameters of the vastus medialis muscle. Clin Anat 2005;18:281-9.

9. Tria AJ Jr. Advancements in minimally invasive total knee arthroplasty. Orthopaedics 2003;26(8 Suppl):859-63.

10. Kim Y-H, Kim J-S, Kim D-Y. Clinical outcome and rate of complications after primary total knee replacement performed with quadriceps-sparing or standard arthotomy. J Bone Joint Surg [Br] 2007;89-B:467-70.

11. Insall JN. Intra-articular surgery for degenerative arthritis of the knee: a report of the work of the late KH Pridie. J Bone Joint Surg [Br] 1967;49-B:211-28.

12. Bose K, Kanagasuntheram R, Osman MBH. Vastus medialis oblique: an anatomic and physiological study. Orthopaedics 1980;3:880-3.

13. Koskinen SK, Kujala UM. Patellofemoral relationships and distal insertion of the vastus medialis muscle: a magnetic resonance imaging study in non symptomatic subjects and in patients with patellar dislocation. Arthroscopy 1992;3:465-8. 